\title{
Evaluation of Head and Neck Cancer Awareness and Screening Status in Jeddah, Saudi Arabia
}

\author{
Turki Y Alhazzazi
}

\begin{abstract}
Background: Head and neck cancer (HNC) is one of the deadliest cancers worldwide. Early detection is a key determinant of HNC prognosis. Hence, raising awareness of this disease may improve survival rates. The present study evaluated the level of awareness and screening status for HNC in the general population of Jeddah, Saudi Arabia. Materials and Methods: An e-questionnaire was distributed during our HNC awareness campaign at the Red Sea Mall in Jeddah. The questions assessed HNC knowledge and screening status among participants. Results: Of the 112 respondents, $68 \%$ indicated that they had no knowledge of HNC. Social media was the major source of information $(39 \%)$ for respondents. The majority $(40 \%)$ believed that it was the joint responsibility of dentists, dental hygienists, and general physicians to screen for $\mathrm{HNC}$; $82 \%$ had never been screened. Conclusions: Knowledge and awareness of HNC must be increased in the general population and among dental health professionals.
\end{abstract}

Keywords: Head and neck cancer - HNC - oral cancer - awareness - screening

Asian Pac J Cancer Prev, 17 (3), 1135-1139

\section{Introduction}

Head and neck cancer (HNC) is the current designation for oral cancer, which includes cancers that originate from the oral and nasal cavities, sinuses, lips, salivary glands, throat, and larynx(http://globocan.iarc.fr, Accessed 7-2015). HNC is the ninth most common cancer worldwide (http://globocan.iarc.fr, Accessed 7-2015), and in Saudi Arabia, it is the third most common cancer after lymphoma and leukemia and as such, represents a significant public health concern (Al-Balawi and Nwoku, 2002). Moreover, the prevalence of HNC is dependent on geographic location; the incidence is highest in the southern region (Al-Ghamdi et al., 1994; Tandon et al., 1995; Al-Balawi and Nwoku, 2002). The 5-year survival rate is poor, averaging around $50 \%$, not changing for decades (http://www.cancer.org/Accessed 7-2015).

Alcohol and tobacco use are the main risk factors for HNC (Galbiatti et al., 2013; http://www.cancer. org/, Accessed 7-2015). However, infection with human papilloma virus (HPV), human immunodeficiency virus (HIV), poor oral hygiene, ill-fitting oral appliances that injure the mucosa, and family history also seem to play a role (Galbiatti et al., 2013; http://www.cancer.org/, Accessed 7-2015), while $25 \%$ of all HNC patients have no obvious risk factors, highlighting the complexity of the disease (http://www.cancer.org/, Accessed 7-2015). Risk factors specific to the population of Saudi Arabia are the use of smokeless tobacco such as shammah and alqat, which are associated with carcinogenesis, especially in the southern region of Gizan (Salem et al., 1984; Ibrahim et al., 1986; Allard et al., 1999; El-Husseiny et al., 2000; Zhang et al., 2001; Alsanosy, 2014).

It is worth noting that not all patients experience pain as part of the disease process (Scharpf et al., 2009), which presents a challenge for early detection; indeed, the widely held misconception in the general population that cancer is associated with pain must be dispelled. The lack of awareness about HNC among patients as well as health professionals can delay diagnosis, referral, and hence treatment. The present study evaluated the level of awareness about HNC as well as screening status in the general population of Jeddah, Saudi Arabia.

\section{Materials and Methods}

An e-questionnaire was developed using Google forms and accessed using tablet devices; it included questions that assessed both knowledge and screening status of HNC in a random sample of the population in Jeddah, Saudi Arabia. The study was conducted during our 3rd HNC awareness campaign in April 2015 at the Red Sea Mall, the largest mall in Jeddah.

The questionnaire was explained to all participants. Written, informed consent was obtained from all participants. The study was approved by the research ethical committee board from King Abdulaziz University, Faculty of Dentistry and Red Sea Mall Administration, and was in full accordance with the World Medical Association Declaration of Helsinki. 


\section{Results}

A total of 400 adults participated in the HNC awareness campaign; of these, 112 individuals (28\%; $40 \%$ male and $60 \%$ female) participated in the study. Most of the participants were $20-30$ years old $(45 \%)$ or $30-40$ years old (28\%) (Figure 1A); $43 \%$ were employed, $27 \%$ were students, and $27 \%$ were housewives (Figure

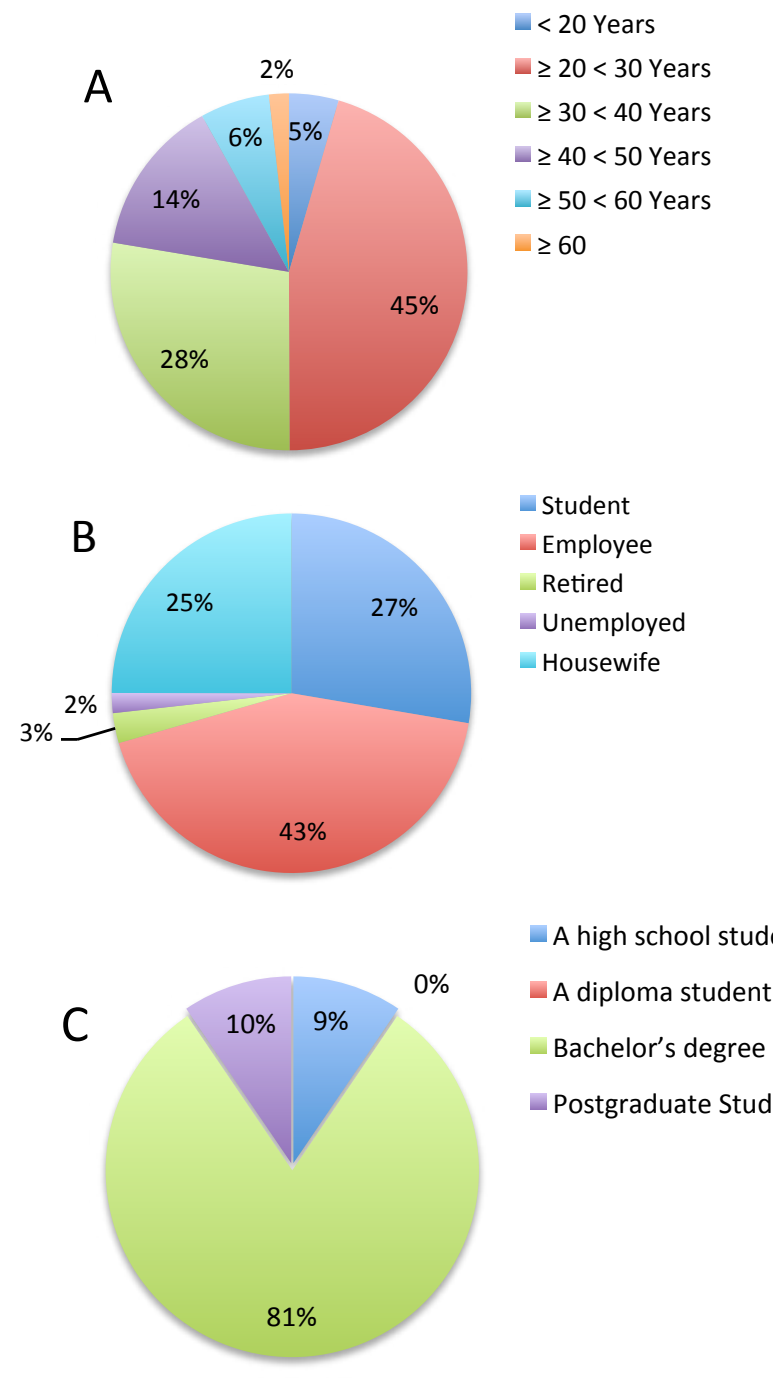

Figure 1. Demographic Data and Education Level of Study Participants. (A) Age distribution. (B) Employment status. (C) Educational qualification of students

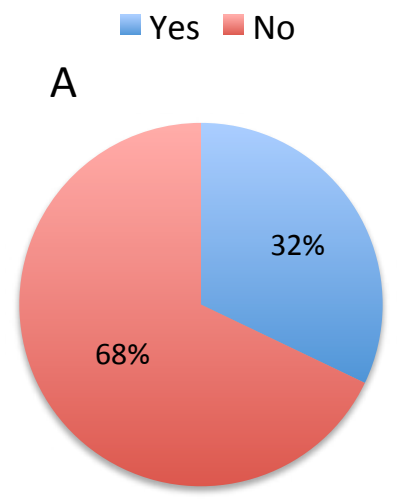

1B). The majority held bachelor degrees, whereas few had post-graduate education (Figure 1C). Unexpectedly, $68 \%$ of visitors indicated that they had never received any information about HNC (Figure 2A); those who had received information (32\%) had obtained it mostly from social media (39\%) or dentists (33\%), through educational lectures (28\%), or from television (28\%) (Figure 2B).
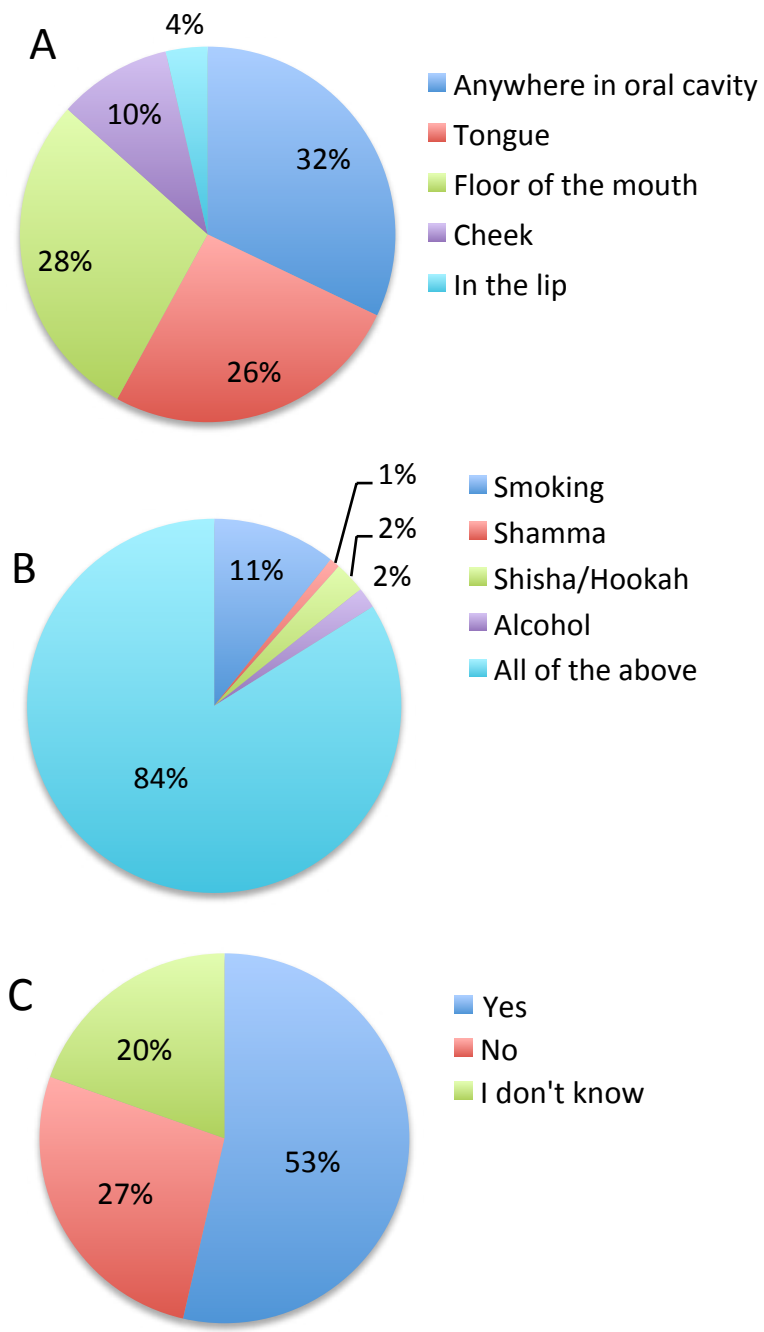

Figure 3. Participants' Knowledge of HNC. (A) Knowledge of common HNC lesion locations. (B) Knowledge of different types of smoking and alcohol as HNC risk factors. (C) Knowledge regarding pain as a characteristic of HNC

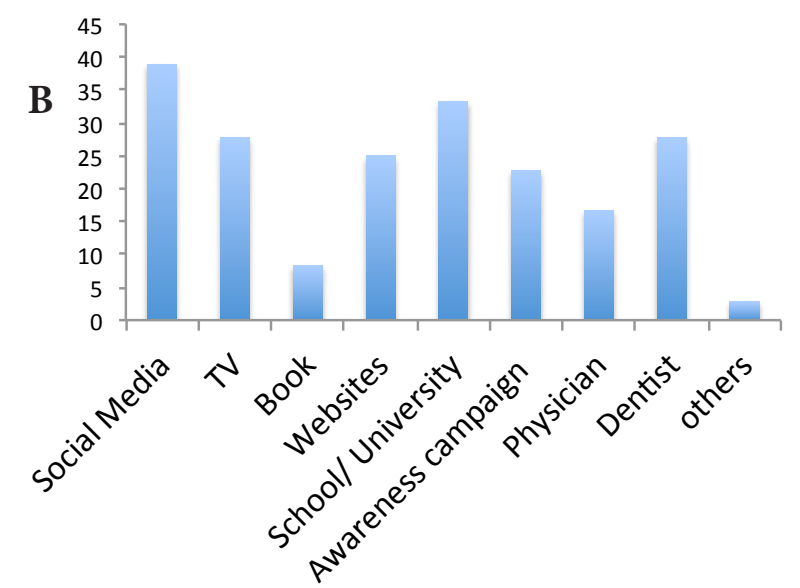

Figure 2. HNC Awareness Level. (A) Knowledge of HNC. (B) Source of information 
A

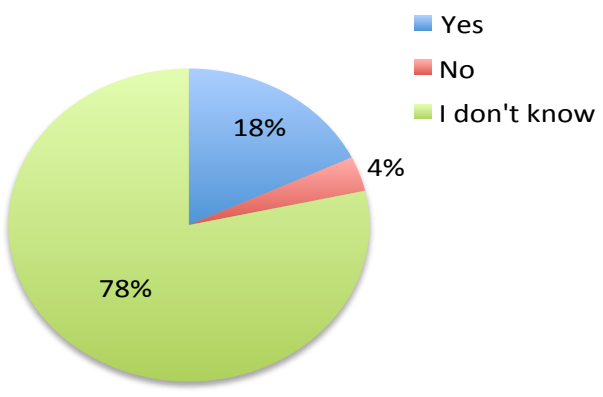

$\mathrm{C}$

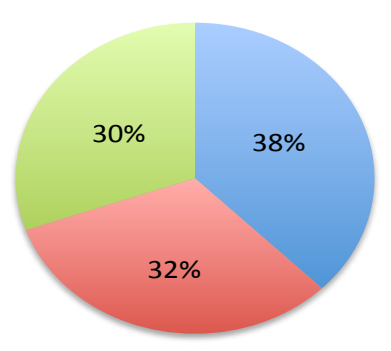

B

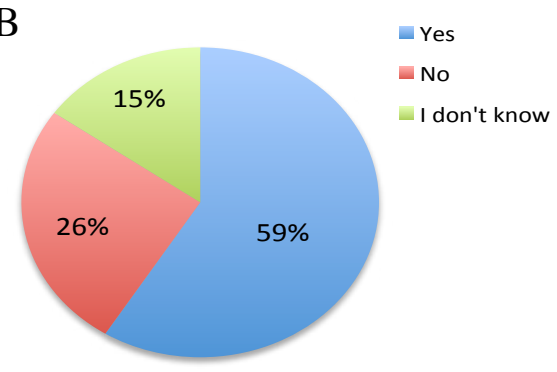

Yes
No
I don't know

$\mathrm{D}$

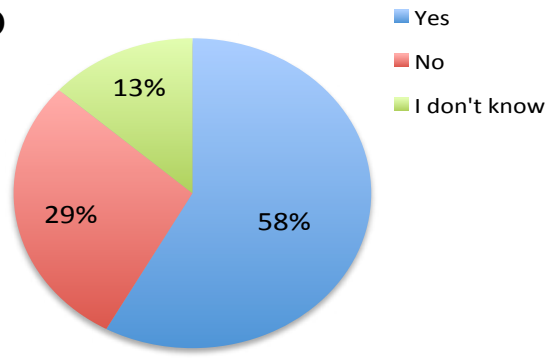

Figure 4. Participants' Knowledge of other Causes of HNC. Role of (A) HPV, (B) HIV, (C) oral health, and (D) genetics

A

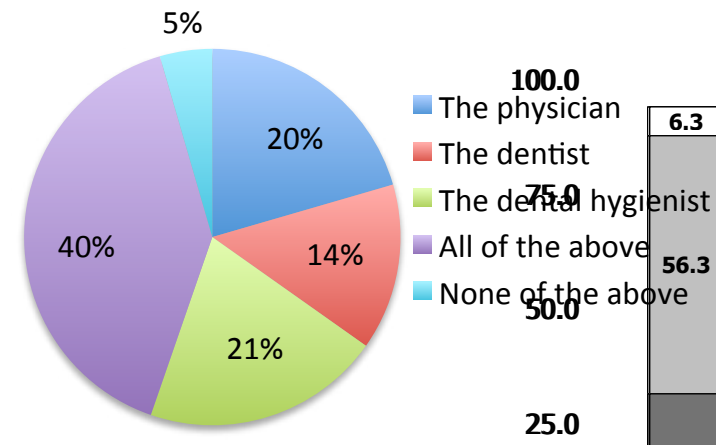

B

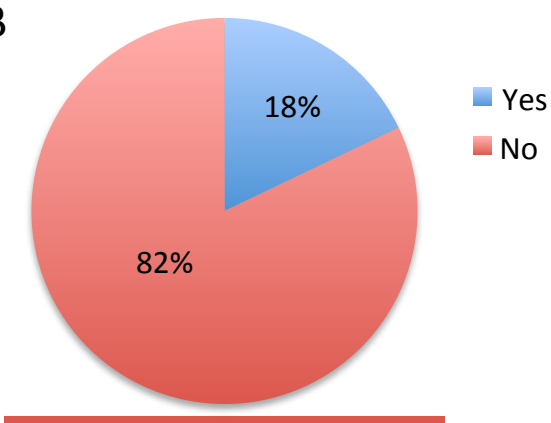

C

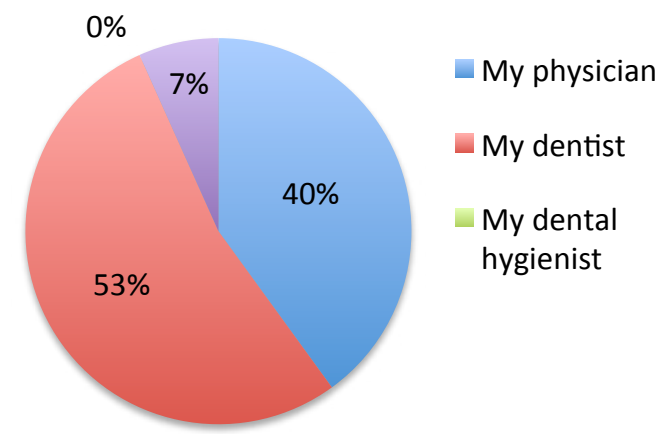

Figure 5. Current Status of Health Care Providers in HNC Screening. (A) Participants' opinion on health care providers' role in screening. (B) Participant screening status for HNC. (C) Percentage of health care providers conducting HNC screening
Only $26 \%$ and $28 \%$ of participants identified the tongue and floor of the mouth, respectively, as the most common locations of HNC, while $32 \%$ thought it could occur anywhere in the oral cavity (Figure 3A). The majority of participants (84\%) correctly identified cigarette smoking, and use of shammah, hoka, and alcohol as $\mathrm{HNC}$ risk factors, while only $10 \%$ identified cigarette smoking alone as a major factor (Figure 3B). In addition, $53 \%$ thought that $\mathrm{HNC}$ is always painful; while the remaining participants either did not know (20\%) or did not think pain was a typical presentation of HNC (27\%) (Figure 3C). Only 19\% of participants had heard of HPV (data not shown), but only $18 \%$ identified it as a risk factor for $\mathrm{HNC}$ (Figure 4A). In contrast, the percentages of individuals who thought or did not think HIV was a risk factor or who did not know were similar (Figure 4B). Interestingly, 59\% of participants cited poor oral hygiene as a risk factor, while $26 \%$ believed it was irrelevant (Figure 4C). Most participants (58\%) thought genetics predisposes to HNC, whereas $28.6 \%$ did not have this belief (Figure 4D).

A large percentage of participants ( $40 \%$ ) believed that screening for $\mathrm{HNC}$ is the joint responsibility of dentists, dental hygienists, and general physicians (Figure 5A); however, $82 \%$ had never been screened for $\mathrm{HNC}$, while the remainder were screened by dentists $(53 \%)$ or physicians (40\%) but not by dental hygienists (Figure 5B).

\section{Discussion}

The results of this study revealed a considerable lack of knowledge and awareness of HNC and associated risk factors among the general population in Jeddah. This is consistent with studies conducted in other countries such as United Arab Emirates, Britain, USA, and Iran among others (West et al., 2006; Amarasinghe et al., 2010; Pakfetrat et al., 2010; O Connor et al., 2010; Al-Rawi et 
al., 2012; Luryi et al., 2014; Dwojak et al., 2015).

Although social media is an effective and accessible way to deliver information and raise public knowledge and awareness of diseases (Saleh et al., 2012), and was one of the major sources of HNC information in the study population, not all registered accounts provide accurate information. As such, there is a need for social media accounts monitored by licensed experts. In addition, dentists who are one of the primary sources of HNC information-should strive to stay current with HNC diagnosis methodologies and ensure that they screen all patients regardless of their specialization.

Most participants (83.9\%) knew that use of tobacco products (including cigarettes, shamma, and hoka), and alcohol are considered risk factors for HNC. This is consistent with previously reported findings in other populations (TE O Connor et al., 2010; Croucher et al., 2011; Al-Rawi et al., 2012; Shakoor et al., 2014), and may be attributed to media campaigns highlighting the association between cancer occurrence and tobacco use.

More than $30 \%$ of all HNC lesions occur in the tongue, followed by the floor of the mouth (Bsoul et al., 2005; http://www.cancer.org/, Accessed 7-2015). The majority of our participants did not identify the tongue as a major site of HNC, with $32.1 \%$ suggesting that it could occur anywhere in the oral cavity. However, in a study carried out in Iran, only $8.8 \%$ of participants reported the tongue and floor of the mouth as the most frequently affected sites (Pakfetrat et al., 2010). This indicates a general lack of awareness about the locations of HNC and underscores a need for increasing public knowledge and awareness and promoting $\mathrm{HNC}$ self-examination.

Only $19 \%$ of participants in the present study were aware of HPV, and only $18 \%$ associated it with HNC. This low percentage is in accordance with the 0.8\%-36\% reported by other studies (Luryi et al., 2014; Dwojak et al., 2015). In contrast, the proportion of individuals who knew that HIV was a risk factor for HNC vs. those who were unaware of this association was similar. The general lack of knowledge and awareness of HPV and HIV in Saudi Arabia can be explained by cultural reticence about discussing sexually transmitted diseases.

Interestingly, $58.9 \%$ of participants identified poor oral hygiene as a risk factor for $\mathrm{HNC}$, in contrast to the results of studies carried out in Sri Lanka (11.4\%) and Ireland (12\%) (TE O Connor et al., 2010). Most participants $(58 \%)$ identified genetics as a risk factor as compared to $9.5 \%$ of participants in a study conducted in Sri Lanka (Amarasinghe et al., 2010).

A cause for significant concern is the fact that the majority of participants $(82.1 \%)$ had never been screened for HNC. Similarly, in the Sri Lankan study, $<5 \%$ had undergone screening (Amarasinghe et al., 2010). In addition, although most participants thought that this could be performed by dentists, dental hygienists, or general physicians (40.2\%), only $14 \%$ preferred dentists. This is in agreement with the Iranian study, in which $72.8 \%$ of participants did not think the oral mucosa should be examined by dentists but $60 \%$ preferred general physicians to perform the screening. This suggests that dentists in Jeddah need to educate their patients about HNC and actively promote and practice HNC screening (Pakfetrat et al., 2010).

In conclusions, There is a need for greater knowledge and awareness among both public and dental professionals regarding $\mathrm{HNC}$ and for more routine screening. In addition, health ministries should take definitive action to increase public awareness of HNC in Saudi Arabia, for instance by using social media to reach to the community. Furthermore, engaging health professional-including dentists and dental hygienists - in HNC awareness campaigns throughout the year can have a significant impact on reducing mortality rates associated with this disease.

\section{Acknowledgements}

The author thank Dr. Arwa Algamdi and Maha Bamehesoun for assisting with data analysis. This study was supported by the Deanship of Scientific Research, King Abdulaziz University, Jeddah, Saudi Arabia.

\section{References}

Al-Balawi SA, Nwoku AL (2002). Management of oral cancer in a tertiary care hospital. Saudi Med J, 23, 156-9.

Al-Ghamdi SA, Malatani T, Kameswaran M, et al (1994). Head and neck cancer in a referral center in Asir region. Annals Saudi Med, 14, 383-6.

Al-Rawi NH, Al-Kawas S, Imad O (2012). Public awareness and attitude toward oral cancer screening in united arab emirates. J Inter Dental Med Res, 5, 149- 54.

Allard WF, DeVol EB, Te OB (1999). Smokeless tobacco (shamma) and oral cancer in Saudi Arabia. Community Dent Oral Epidemiol, 27, 398-405.

Alsanosy RM (2014). Smokeless tobacco (shammah) in Saudi Arabia: a review of its pattern of use, prevalence, and potential role in oral cancer. Asian Pac J Cancer Prev, 15, 6477-83.

Amarasinghe HK, Usgodaarachchi US, Johnson NW, et al (2010). Public awareness of oral cancer, of oral potentially malignant disorders and of their risk factors in some rural populations in Sri Lanka. Community Dent Oral Epidemiol, 38, 540-8.

Bsoul SA, Huber MA, Terezhalmy GT (2005). Squamous cell carcinoma of the oral tissues: a comprehensive review for oral healthcare providers. J Contemp Dent Pract, 6, 1-16.

Croucher R, Islam SS, Nunn H (2011). Campaign awareness and oral cancer knowledge in UK resident adult Bangladeshi: a cross-sectional study. Br J Cancer, 105, 925-30.

Dwojak S, Deschler D, Sargent M, et al (2015). Knowledge and screening of head and neck cancer among american indians in South dakota. Am J Public Health, 105, 1155-60.

El-Husseiny G, Kandil A, Jamshed A, et al (2000). Squamous cell carcinoma of the oral tongue: an analysis of prognostic factors. Br J Oral Maxillofac Surg, 38, 193-9.

Galbiatti ALS, Padovani-Junior JA, Maníglia JV, et al (2013). Head and neck cancer: causes, prevention and treatment. Braz J Otorhinolaryngol, 79, 239-47.

http://globocan.iarc.fr (Accessed 7-2015).

http://www.cancer.org/ (Accessed 7-2015).

Ibrahim EM, Satti MB, Al Idrissi HY, et al (1986). Oral cancer in Saudi Arabia: the role of alqat and alshammah. Cancer Detection Prev, 9, 215-8.

Luryi AL, Yarbrough WG, Niccolai LM, et al (2014). Public awareness of head and neck cancers: a cross-sectional survey. 
JAMA Otolaryngol Head Neck Surg, 140, 639-46.

Pakfetrat A, Falaki F, Esmaily HO, et al (2010). Oral cancer knowledge among patients referred to Mashhad Dental School, Iran. Archives Iranian Med, 13, 543- 8.

Saleh A, Yang Y-H, Ghani WMNWA, et al (2012). Promoting oral cancer awareness and early detection using a mass media approach. Asian Pac J Cancer Prev, 13, 1217-24.

Salem G, Juhl R, Schiodt T (1984). Oral malignant and premalignant changes in 'Shammah'-users from the Gizan region, Saudi Arabia. Acta Odontol Scand, 42, 41-5.

Scharpf J, Karnell LH, Christensen AJ, et al (2009). The role of pain in head and neck cancer recurrence and survivorship. Arch Otolaryngol Head Neck Surg, 135, 789-94.

Shakoor A, Rasheed K, Khan YH (2014). Awareness and knowledge about oral cancer in adult patients visiting Islamic International Dental Hospital, Islamabad, Pakistan. Pakistan Oral Dental J, 34, 113- 7.

Tandon P, Pathak VP, Zaheer A, et al (1995). Cancer in the Gizan province of Saudi Arabia: An eleven year study. Ann Saudi Med, 15, 14-20.

TE O Connor, V Papanikolaou, Keogh I (2010). Public knowledge of head and neck cancer. Irish Med J, 103, 105-7.

West R, Alkhatib MN, McNeill A, et al (2006). Awareness of mouth cancer in Great Britain. Br Dent J, 200, 167-9, discussion 51.

Zhang X, Schmitz W, Gelderblom HR, et al (2001). Shammahinduced oral leukoplakia-like lesions. Oral Oncol, 37, 609-12. 